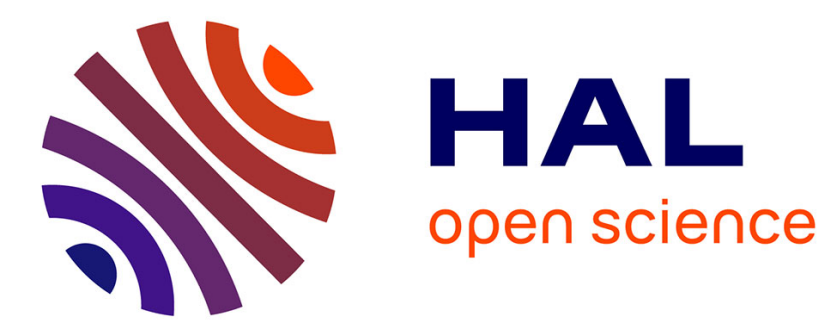

\title{
AN EFFICIENT TRANSMISSION OPERATOR FOR COMPUTING WAVE PROPAGATION BY DOMAIN DECOMPOSITION
}

Denis Duhamel

\section{- To cite this version:}

Denis Duhamel. AN EFFICIENT TRANSMISSION OPERATOR FOR COMPUTING WAVE PROPAGATION BY DOMAIN DECOMPOSITION. Compdyn 2019, Jun 2019, Crète, Greece. hal02915353

\section{HAL Id: hal-02915353 \\ https://hal.science/hal-02915353}

Submitted on 14 Aug 2020

HAL is a multi-disciplinary open access archive for the deposit and dissemination of scientific research documents, whether they are published or not. The documents may come from teaching and research institutions in France or abroad, or from public or private research centers.
L'archive ouverte pluridisciplinaire HAL, est destinée au dépôt et à la diffusion de documents scientifiques de niveau recherche, publiés ou non, émanant des établissements d'enseignement et de recherche français ou étrangers, des laboratoires publics ou privés. 


\title{
AN EFFICIENT TRANSMISSION OPERATOR FOR COMPUTING WAVE PROPAGATION BY DOMAIN DECOMPOSITION
}

\author{
Denis Duhamel ${ }^{1}$ \\ ${ }^{1}$ Ecole des Ponts ParisTech \\ Laboratoire Navier, Champs sur Marne, France \\ e-mail: denis.duhamel@enpc.fr
}

Keywords: Domain decomposition, wave, Helmholtz, finite element, transmission operator.

\begin{abstract}
For large size problems, domain decompositions can be used to solve wave propagation problems such as the Helmholtz equation. Generally, this leads to an iterative process where data are exchanged at the boundary between subdomains. Depending on the quality of this exchange the number of iterations is more or less. Moreover, this number of iterations can depend on the frequency and on the number of domains. Here, we propose transmission operators approximating the Dirichlet to Neumann (DtN) operator which is known to be near optimal. We show this can be done using only the solution of problems involving sparse matrices and so keeping the computational time at an acceptable level. When this is combined with the double sweep preconditioner and that the computational domain is decomposed into a sequel of slices this results in an algorithm with a low number of iterations. Different examples are presented to support the precedent analysis.
\end{abstract}




\section{INTRODUCTION}

Solving wave propagation problems described by the Helmholtz equation is important in many domains such as acoustics or elastic wave propagation. This can be difficult as the frequency increases because fine meshes have to be used in these cases. One possibility to increase the computing power is to use domain decompositions for which the domain is decomposed into subdomains on which the solutions can be computed more easily by solving small size problems. This generally involves an iterative scheme with a communication between the subdomains at each step, for instance by the transmission of data across the boundary of subdomains.

Many works have been done in the past on this subject where we find methods in which the subdomains overlap or not. In the field of non-overlapping methods we find the works on FETI methods as in [1] or for the application to the Helmholtz equation in [2] where interdomains fields continuity is obtained by Lagrange multipliers. These Lagrange multipliers are computed by solving a relatively small dual problem by a preconditioned conjugate gradient algorithm.

Another possibility is to use overlapping or non-overlapping Schwartz methods as in [3, 4, 5] where the solution is computed iteratively in each subdomain and transmission conditions are defined at the boundary of each domain from the solutions in the subdomains at the precedent step. The convergence rate mainly depends on this transmission operator at the boundary, the best one being the DtN map, see [6, 7], but it is too complicated to compute for being usable. Different methods have been proposed to approximate this complex non-local operator by a simpler one, often by local operators on the boundary, see for instance [8] and [9] for an uptodate view on the subject. A PML layer can also be used as in [10].

Here we propose a new transmission operator defined from the solution of a wave problem in domains around the computed subdomain as in the PML but with a simpler formulation which does not need modifications to the problem formulation or computations of new stiffness matrices. For being efficient, a preconditionner must be used with these methods such as the double sweep preconditionner found in [11, 12, 13].

In section 2, the method is described for the case of a problem posed on two half planes where analytical results can be found following the approach of [5] but with a new transmission operator, then this is applied to more general domains which can be decomposed into a sequel of slices. Finally, in section 3, numerical results are presented before a conclusion.

\section{COMPUTATION BY DOMAIN DECOMPOSITION}

The objective is to solve a wave propagation problem by domain decomposition. We consider here the case of the Helmholtz equation given by

$$
\Delta p+k^{2} p=0
$$

with $p$ the pressure, $k=\omega / c$ the wavenumber, $c$ the sound velocity, $\omega=2 \pi f$ and $f$ the frequency. This is defined on a domain $\Omega$ with a Neumann boundary condition as $\frac{\partial p}{\partial n}=q$ on the boundary $\Gamma$ of $\Omega$.

\subsection{Case of two half-planes}

Consider first the Helmholtz equation defined on a plane $(x, y)$ which is divided into two halfplane subdomains by the line $x=0$, see figure 1. Taking the Fourrier transform in the $y$ variable with the wavenumber $k_{y}$, one obtains the following sequel of one-dimensional problems in the 


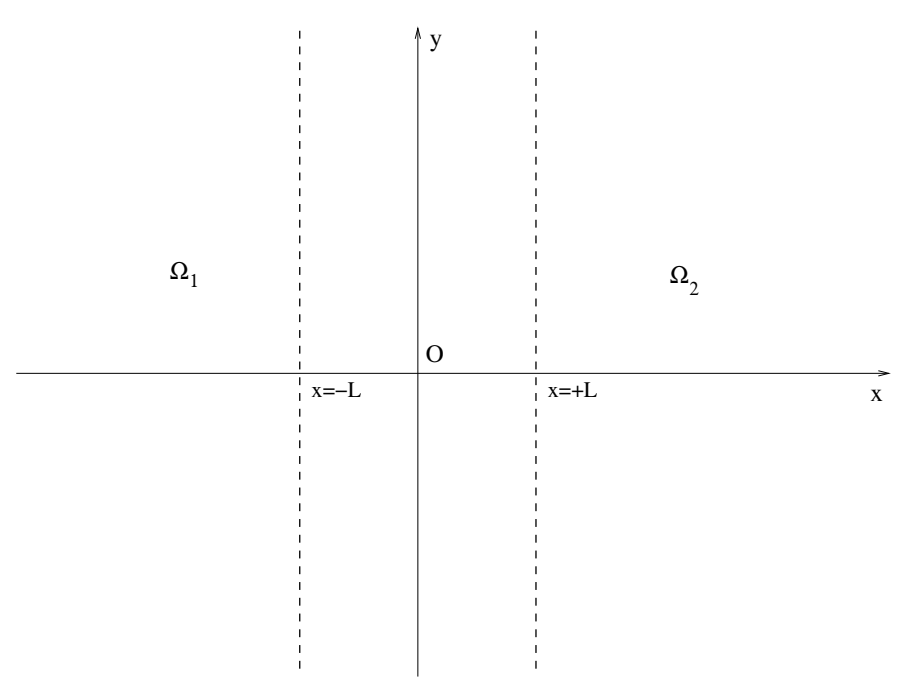

Figure 1: Two half-planes with additional slices of thickness L.

two sub-domains, which are respectively

$$
\begin{aligned}
\frac{\partial^{2} p_{1}^{n+1}}{\partial x^{2}}+\left(k^{2}-k_{y}^{2}\right) p_{1}^{(n+1)} & =0 \text { for } x<0 \\
\frac{\partial p_{1}^{(n+1)}}{\partial x}+\sigma_{1}\left(k_{y}\right) p_{1}^{(n+1)} & =\frac{\partial p_{2}^{(n)}}{\partial x}+\sigma_{1}\left(k_{y}\right) p_{2}^{(n)} \text { at } x=0 \\
\frac{\partial^{2} p_{2}^{(n+1)}}{\partial x^{2}}+\left(k^{2}-k_{y}^{2}\right) p_{2}^{(n+1)} & =0 \text { for } x>0 \\
-\frac{\partial p_{2}^{(n+1)}}{\partial x}+\sigma_{2}\left(k_{y}\right) p_{2}^{(n+1)} & =-\frac{\partial p_{1}^{(n)}}{\partial x}+\sigma_{2}\left(k_{y}\right) p_{1}^{(n)} \text { at } x=0
\end{aligned}
$$

with $\sigma_{1}\left(k_{y}\right)$ and $\sigma_{2}\left(k_{y}\right)$ two well chosen functions to assure the convergence of the iterative process. The solutions are respectively

$$
\begin{aligned}
& p_{1}^{(n+1)}(x)=e^{-\lambda\left(k_{y}\right) x} p_{1}^{(n+1)}(0) \text { for } x<0 \\
& p_{2}^{(n+1)}(x)=e^{\lambda\left(k_{y}\right) x} p_{2}^{(n+1)}(0) \text { for } x>0
\end{aligned}
$$

with $\lambda\left(k_{y}\right)=i \sqrt{k^{2}-k_{y}^{2}}$. From the transmission conditions (second lines of 2 and 3 , one gets

$$
\begin{aligned}
p_{1}^{(n+1)}(0) & =\frac{\lambda\left(k_{y}\right)+\sigma_{1}\left(k_{y}\right)}{-\lambda\left(k_{y}\right)+\sigma_{1}\left(k_{y}\right)} p_{2}^{(n)}(0) \\
p_{2}^{(n+1)}(0) & =\frac{\lambda\left(k_{y}\right)+\sigma_{2}\left(k_{y}\right)}{-\lambda\left(k_{y}\right)+\sigma_{2}\left(k_{y}\right)} p_{1}^{(n)}(0)
\end{aligned}
$$

so that

$$
p_{1}^{(2 n)}(0)=\rho^{n}\left(k_{y}\right) p_{1}^{(0)}(0)
$$

with

$$
\rho\left(k_{y}\right)=\frac{\lambda\left(k_{y}\right)+\sigma_{1}\left(k_{y}\right)}{-\lambda\left(k_{y}\right)+\sigma_{1}\left(k_{y}\right)} \cdot \frac{\lambda\left(k_{y}\right)+\sigma_{2}\left(k_{y}\right)}{-\lambda\left(k_{y}\right)+\sigma_{2}\left(k_{y}\right)}
$$


Different possibilities for choosing the values of $\sigma_{1}$ and $\sigma_{2}$ are discussed in [5]. Here, we propose another way of building these functions. The value of $\sigma_{1}\left(k_{y}\right)$ is obtained from the solution of the problem in the slice $0<x<L$ with absorbing boundary conditions at $x=L$, more precisely from the solution of the problem

$$
\begin{array}{rll}
\frac{\partial^{2} p_{1}}{\partial x^{2}}+\left(k^{2}-k_{y}^{2}\right) p_{1} & =0 & \text { for } 0<x<L \\
p_{1} & =p & \text { for } x=0 \\
\frac{\partial p_{1}}{\partial x}-\gamma_{1}\left(k_{y}\right) p_{1} & =0 & \text { for } x=L
\end{array}
$$

and $\sigma_{1}\left(k_{y}\right)$ is defined such that $\frac{\partial p_{1}}{\partial x}(0)+\sigma_{1}\left(k_{y}\right) p=0$. The solution of problem 8 is given by

$$
p_{1}(x)=a e^{\lambda\left(k_{y}\right) x}+b e^{-\lambda\left(k_{y}\right) x}
$$

with the coefficients $a$ and $b$ such that

$$
\begin{aligned}
a+b & =p \\
\lambda\left(k_{y}\right)\left(a e^{\lambda\left(k_{y}\right) L}-b e^{-\lambda\left(k_{y}\right) L}\right)-\gamma_{1}\left(k_{y}\right)\left(a e^{\lambda\left(k_{y}\right) L}+b e^{-\lambda\left(k_{y}\right) L}\right) & =0
\end{aligned}
$$

The second relation of 10 leads to $b=R_{1} a$ with $R_{1}=\frac{\lambda\left(k_{y}\right)-\gamma_{1}\left(k_{y}\right)}{\lambda\left(k_{y}\right)+\gamma_{1}\left(k_{y}\right)} e^{2 \lambda\left(k_{y}\right) L}$, so that we get

$$
\sigma_{1}\left(k_{y}\right)=-\lambda\left(k_{y}\right) \frac{1-R_{1}}{1+R_{1}}
$$

Similarly one gets

$$
\sigma_{2}\left(k_{y}\right)=-\lambda\left(k_{y}\right) \frac{1-R_{2}}{1+R_{2}}
$$

In the case $k_{y}=0$, taking $\gamma_{1}=\gamma_{2}=i k$, one gets $R_{1}=R_{2}=R$ and the classical values $\sigma_{1}=$ $\sigma_{2}=-i k$. From (11) and (12) we obtain the value of $\rho\left(k_{y}\right)$ by relation 7 giving $\rho\left(k_{y}\right)=R^{2}$ in the case $\gamma_{1}=\gamma_{2}=i k$. The function $\rho\left(k_{y}\right)$ is plotted versus $k_{y} / k$ for several values of $k L$ in figure 2. For $k L=0$, the evanescent waves are not attenuated and the process cannot converge. On the contrary, for $k L>0$, the value of $\rho\left(k_{y}\right)$ is lower than one for the entire spectrum leading to the convergence of the iterative process and all the more so as the value of $k L$ is high.

\subsection{General method}

We consider now a more general domain which is decomposed into vertical slices as shown in figure 3 . In each vertical band $i$, one has to solve at step $n+1$

$$
\begin{aligned}
\Delta p_{i}^{(n+1)}+k^{2} p_{i}^{(n+1)} & =0 \text { in } \Omega_{i} \\
\frac{\partial p_{i}^{(n+1)}}{\partial n_{i}}+S_{i, i-1} p_{i}^{(n+1)} & =g_{i, i-1}^{(n)}=-\frac{\partial p_{i-1}^{(n)}}{\partial n_{i-1}}+S_{i, i-1} p_{i-1}^{(n)} \text { on } \Gamma_{i-1, i} \\
\frac{\partial p_{i}^{(n+1)}}{\partial n_{i}}+S_{i, i+1} p_{i}^{(n+1)} & =g_{i, i+1}^{(n)}=-\frac{\partial p_{i+1}^{(n)}}{\partial n_{i+1}}+S_{i, i+1} p_{i+1}^{(n)} \text { on } \Gamma_{i, i+1}
\end{aligned}
$$

with the operators $S$ as approximations of the DtN operators on the boundaries and the $g_{i, i+1}$ are updated by

$$
\begin{aligned}
g_{i, i+1}^{(n+1)} & =-\frac{\partial p_{i+1}^{(n+1)}}{\partial n_{i+1}}+S_{i, i+1} p_{i+1}^{(n+1)} \\
& =-g_{i+1, i}^{(n)}+\left(S_{i, i+1}+S_{i+1, i}\right) p_{i+1}^{(n+1)}
\end{aligned}
$$




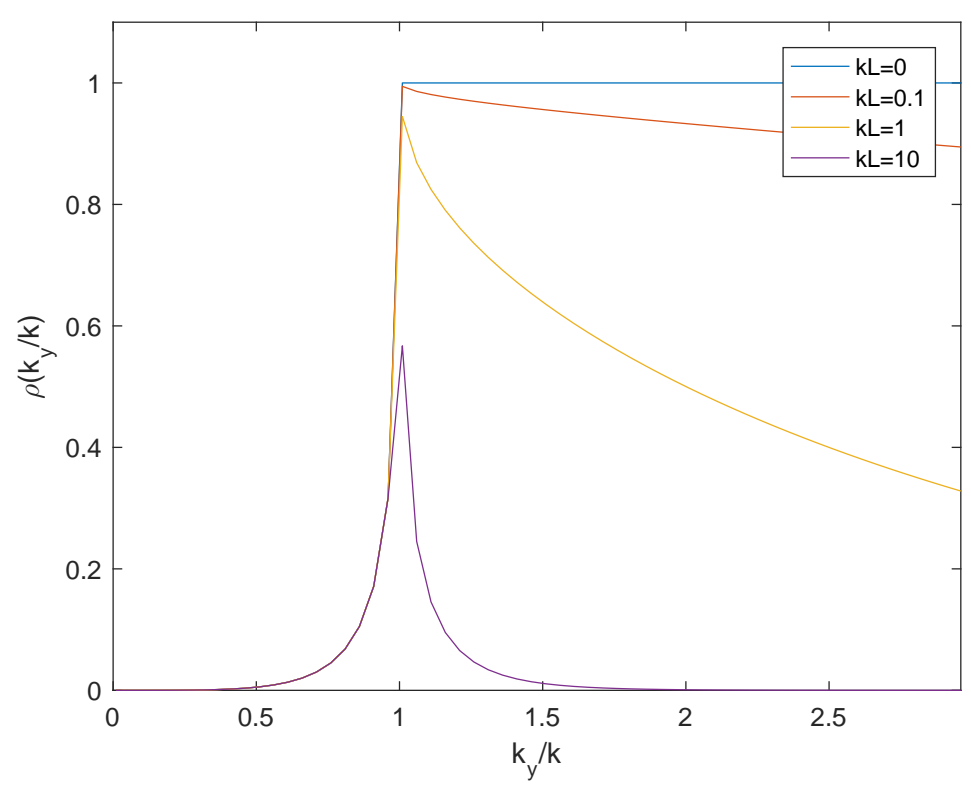

Figure 2: Values of $\rho$ for different $k L$

with a similar formula for $g_{i, i-1}^{(n+1)}$.

The operator $S_{i, i+1} p_{i+1}^{(n+1)}$ is obtained by solving the Helmholtz equation in the domain $\Omega_{i+1}$ with an absorbing boundary condition on $\Gamma_{i+1, i+2}$, that is

$$
\begin{aligned}
\Delta u+k^{2} u & =0 \text { in } \Omega_{i+1} \\
\frac{\partial u}{\partial n_{i+1}}-i k u+\frac{i}{4 k} \frac{\partial^{2} u}{\partial \tau^{2}} & =0 \text { on } \Gamma_{i+1, i+2} \\
u & =p_{i+1}^{(n+1)} \text { on } \Gamma_{i, i+1}
\end{aligned}
$$

and $S_{i, i+1} p_{i+1}^{(n+1)}=\frac{\partial u}{\partial n_{i+1}}$ on $\Gamma_{i, i+1}$ giving an approximation of the DtN operator on that interface.

The operator $S_{i+1, i} p_{i+1}^{(n+1)}$ is obtained by solving the Helmholtz equation in the domain $\Omega_{i}$ with an absorbing boundary condition on $\Gamma_{i-1, i}$, that is

$$
\begin{aligned}
\Delta u+k^{2} u & =0 \text { in } \Omega_{i} \\
\frac{\partial u}{\partial n_{i}}-i k u+\frac{i}{4 k} \frac{\partial^{2} u}{\partial \tau^{2}} & =0 \text { on } \Gamma_{i-1, i} \\
u & =p_{i+1}^{(n+1)} \text { on } \Gamma_{i, i+1}
\end{aligned}
$$

with $\frac{\partial}{\partial \tau}$ the tangential derivative and $S_{i+1, i} p_{i+1}^{(n+1)}=\frac{\partial u}{\partial n_{i}}$ on $\Gamma_{i, i+1}$ giving an approximation of the DtN operator on that interface. 


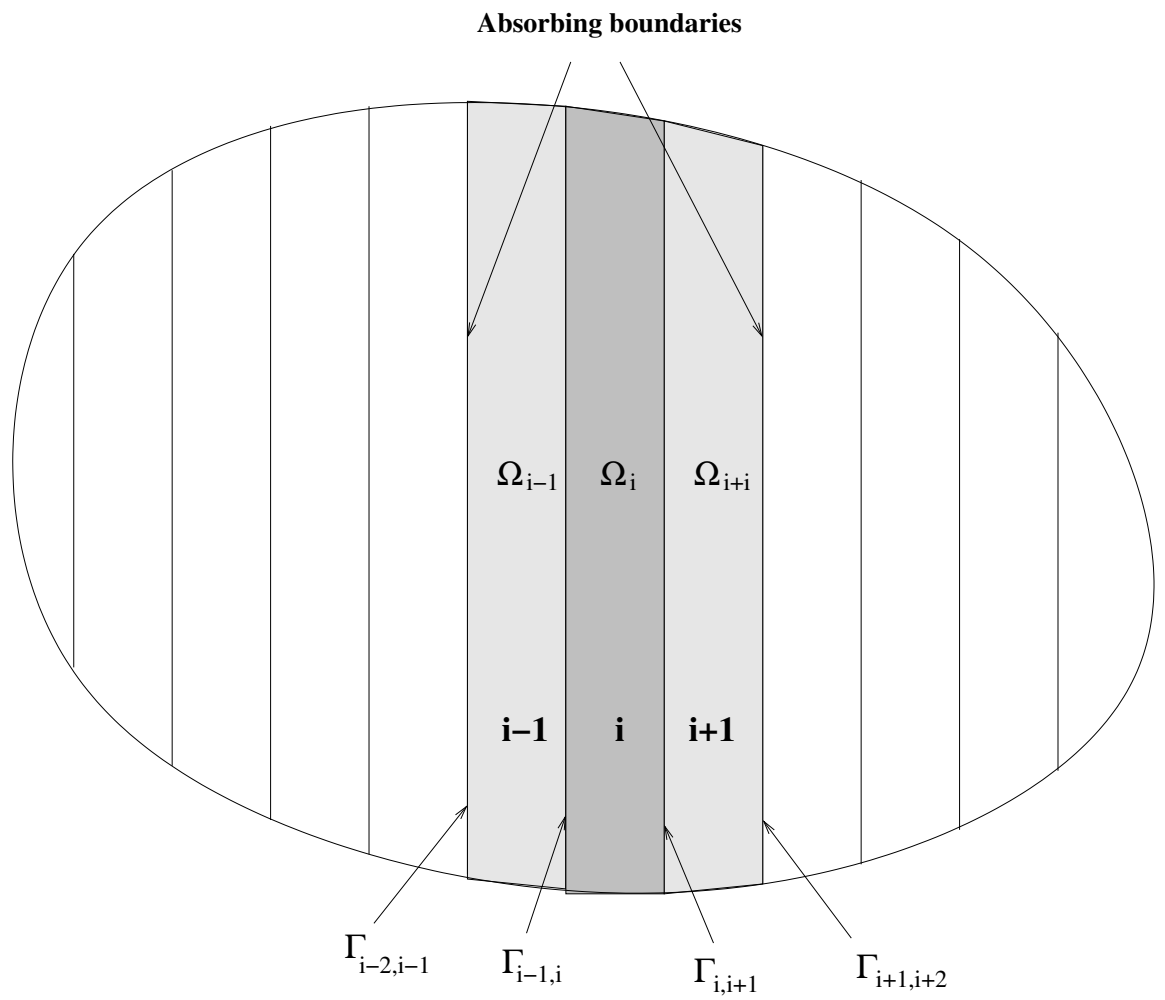

Figure 3: Domain and its decomposition into subdomains

Combining relations 13,15 and 16 , to update the solution in $\Omega_{i}$, one has to solve

$$
\begin{aligned}
\Delta p_{i}^{(n+1)}+k^{2} p_{i}^{(n+1)} & =0 \text { in } \Omega_{i} \\
\Delta u_{i-1}^{(n+1)}+k^{2} u_{i-1}^{(n+1)} & =0 \text { in } \Omega_{i-1} \\
\Delta u_{i+1}^{(n+1)}+k^{2} u_{i+1}^{(n+1)} & =0 \text { in } \Omega_{i+1} \\
\frac{\partial p_{i}^{(n+1)}}{\partial n_{i}}+\frac{\partial u_{i-1}^{(n+1)}}{\partial n_{i-1}} & =g_{i, i-1}^{(n)} \text { on } \Gamma_{i-1, i} \\
\frac{\partial p_{i}^{(n+1)}}{\partial n_{i}}+\frac{\partial u_{i+1}^{(n+1)}}{\partial n_{i+1}} & =g_{i, i+1}^{(n)} \text { on } \Gamma_{i, i+1} \\
\frac{\partial u_{i-1}^{(n+1)}}{\partial n_{i-1}}-i k u_{i-1}^{(n+1)}+\frac{i}{4 k} \frac{\partial^{2} u_{i-1}^{(n+1)}}{\partial \tau^{2}} & =0 \text { on } \Gamma_{i-2, i-1} \\
\frac{\partial u_{i+1}^{(n+1)}}{\partial n_{i+1}}-i k u_{i+1}^{(n+1)}+\frac{i}{4 k} \frac{\partial^{2} u_{i+1}^{(n+1)}}{\partial \tau^{2}} & =0 \text { on } \Gamma_{i+1, i+2}
\end{aligned}
$$

The last equations of (15) and (16) provide the relations of continuity between the solutions in the adjacent domains. At the discrete level this problem is solved by assembling the dynamic stiffness matrices of the domains $\Omega_{i-1}, \Omega_{i}$ and $\Omega_{i+1}$ and putting forces on the interfaces $\Gamma_{i-1, i}$ and $\Gamma_{i, i+1}$ defined respectively by the functions $g_{i, i-1}^{(n)}$ and $g_{i, i+1}^{(n)}$. For external boundaries, the boundary condition of the problem should be used, for instance $\frac{\partial p}{\partial n}=q_{0}$ for a Neumann boundary defined by a function $q_{0}$.

The problem is then solved by the GMRES algorithm associated to the double sweep preconditionner described in [11, 12, 13]. For the updating of $g_{i, i+1}$, one has to solve the problems 
(15) and (16) and use their solutions in (14).

\section{NUMERICAL EXAMPLES}

\subsection{Rectangle}

We first consider the case of a rectangular domain of size $1 m \times 1 m$ with a boundary condition given by a plane wave $e^{i k x}$ on the left and right boundaries as $\frac{\partial p}{\partial n}=i k n_{x} e^{i k x}$. The mesh is created with triangular elements of degree 2 (52633 nodes for 5 subdomains up to 80401 nodes for 100 subdomains). The mesh for 100 subdomains is shown on figure 4 where each domain is a fine vertical band. Computations are done for different frequencies and number of subdomains. Table 1 shows that in this case the number of iterations is very low and do not depend on the frequency or the number of subdomains. The solution for 100 subdomains and the frequency $2000 \mathrm{~Hz}$ is shown on the right of figure 5 .

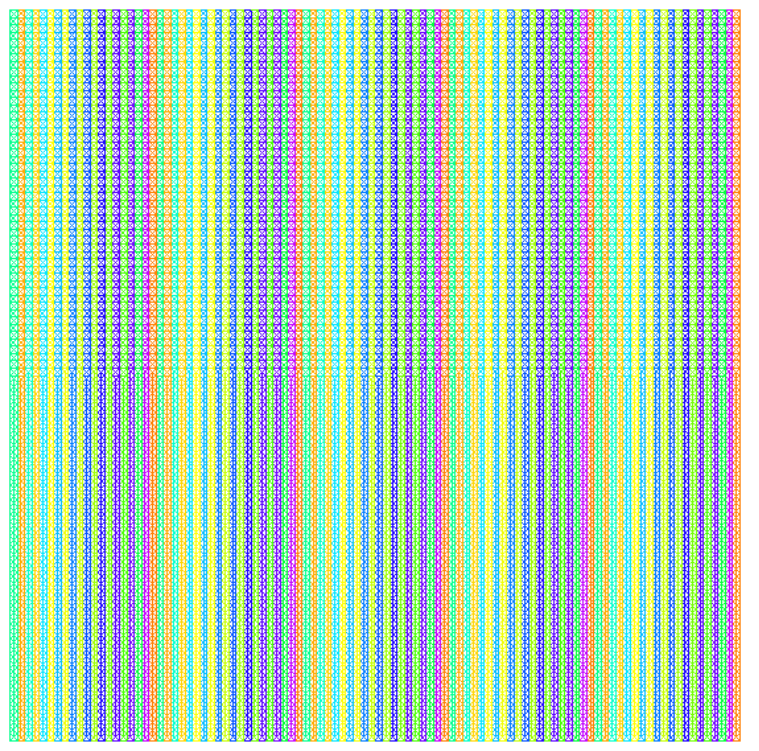

Figure 4: Rectangular domain with 100 subdomains

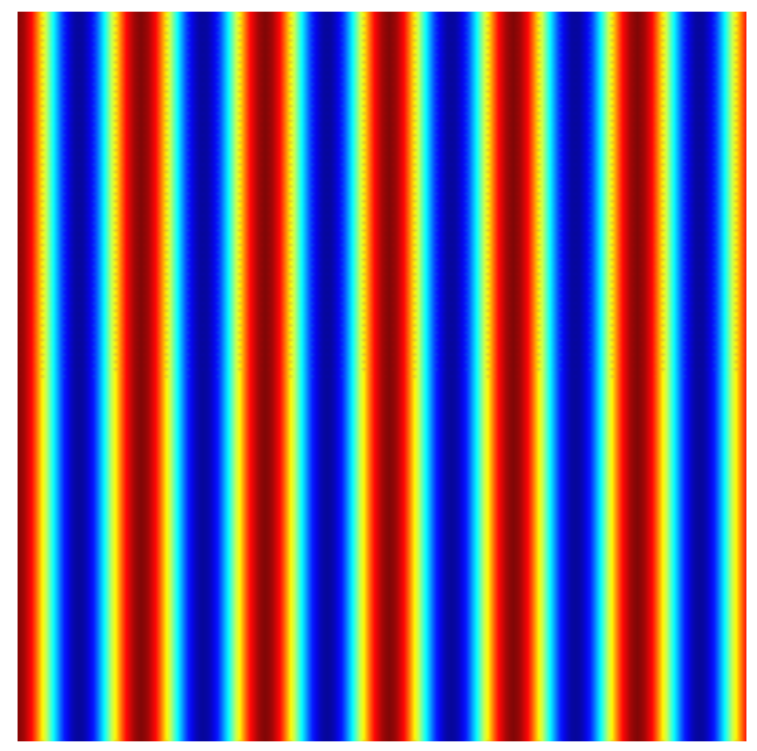

Figure 5: Solution at $2000 \mathrm{~Hz}$ for 100 subdomains

Other computations are made with the boundary condition given by $\cos \left(k_{y} y\right) e^{i k_{x} x}$ with $k_{y}=$ $4 \pi / L_{y}$ and $k_{x}=\sqrt{k^{2}-k_{y}^{2}}$. The number of iteration is given in table 2 while the solutions for $500 \mathrm{~Hz}$ and $2000 \mathrm{~Hz}$ are given in figures 6 and 7 . In figure 6 the boundary condition leads to an evanescent wave while in figure 6 a propagating wave is clearly visible. This number of iterations is low except for 100 subdomains and low frequencies. This needs further studies to understand this phenomenon as usually the number of iteration is increasing with the frequency.

\begin{tabular}{cccc}
\hline Frequency & \multicolumn{2}{c}{ Number of subdomains } \\
& 5 & 25 & 100 \\
\hline $500 \mathrm{~Hz}$ & 4 & 4 & 4 \\
$1000 \mathrm{~Hz}$ & 4 & 4 & 4 \\
$2000 \mathrm{~Hz}$ & 4 & 4 & 4 \\
\hline
\end{tabular}

Table 1: Number of iterations for different frequencies and number of subdomains. 


\begin{tabular}{cccc}
\hline Frequency & \multicolumn{3}{c}{ Number of subdomains } \\
& 5 & 25 & 100 \\
\hline $500 \mathrm{~Hz}$ & 10 & 15 & 73 \\
$1000 \mathrm{~Hz}$ & 13 & 15 & 41 \\
$2000 \mathrm{~Hz}$ & 17 & 18 & 20 \\
\hline
\end{tabular}

Table 2: Number of iterations for different frequencies and number of subdomains.

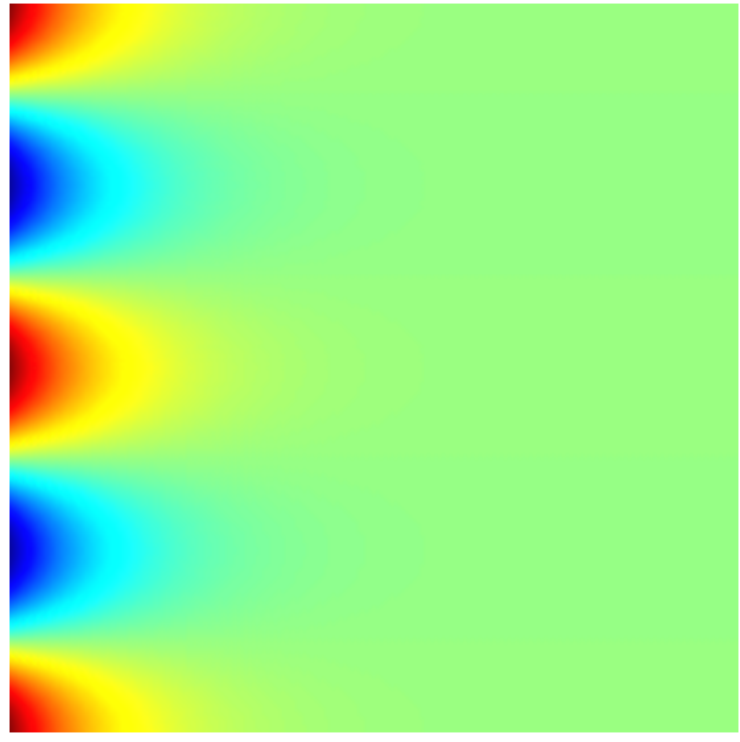

Figure 6: Solution at $500 \mathrm{~Hz}$ for 100 subdomains

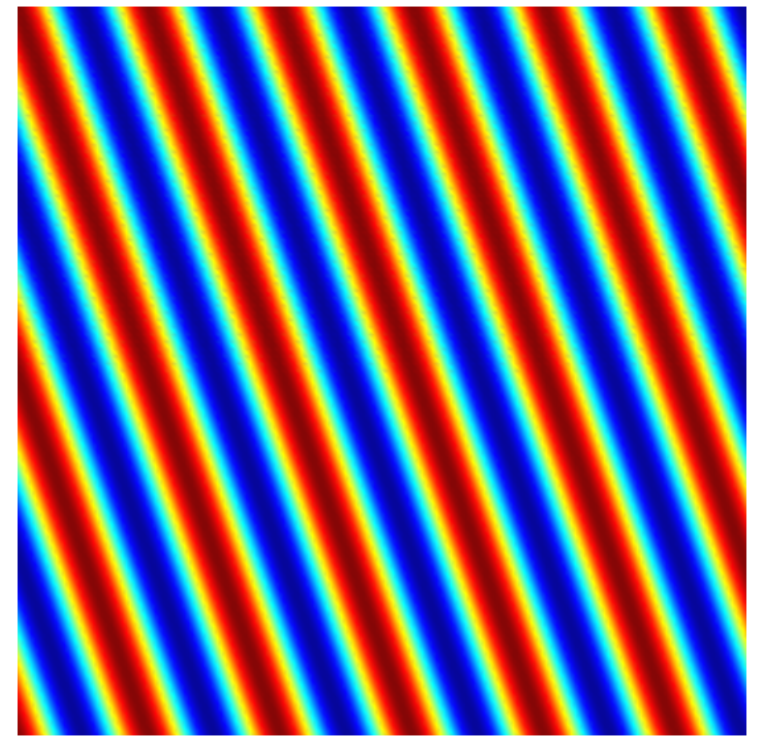

Figure 7: Solution at $2000 \mathrm{~Hz}$ for 100 subdomains

\subsection{Disk}

The domain is now a disk of radius $1 \mathrm{~m}$ whose mesh and decomposition into 100 subdomains is shown in figure 8 . The solution for the plane wave excitation is shown in figure 9 . In this case the number of iterations is shown in table 3 . There is a slow increase in the number of iteration with the frequency and the number of subdomains.

\begin{tabular}{cccc}
\hline Frequency & & Number of subdomains & \\
& 5 & 25 & 100 \\
\hline $500 \mathrm{~Hz}$ & 10 & 14 & 26 \\
$1000 \mathrm{~Hz}$ & 15 & 17 & 23 \\
$2000 \mathrm{~Hz}$ & 28 & 34 & 38 \\
\hline
\end{tabular}

Table 3: Number of iterations for different frequencies and number of subdomains.

\section{CONCLUSION}

We have presented transmission conditions which are intermediate between the local conditions on the boundary and domain conditions as provided by a PML layer. So it gives an equilibrium between the ease of implementation and the efficiency. It involves only modified 


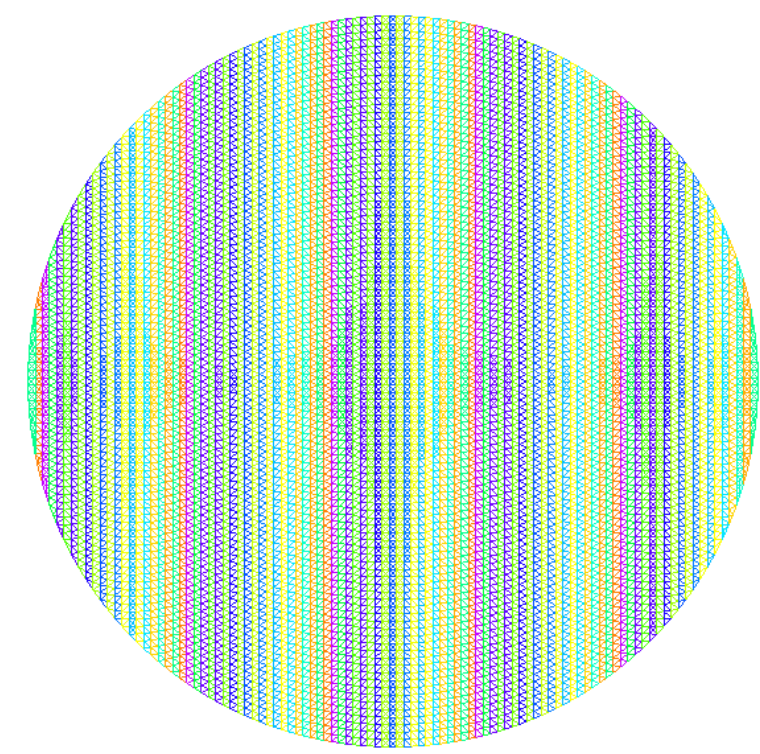

Figure 8: Disk domain with 100 subdomains

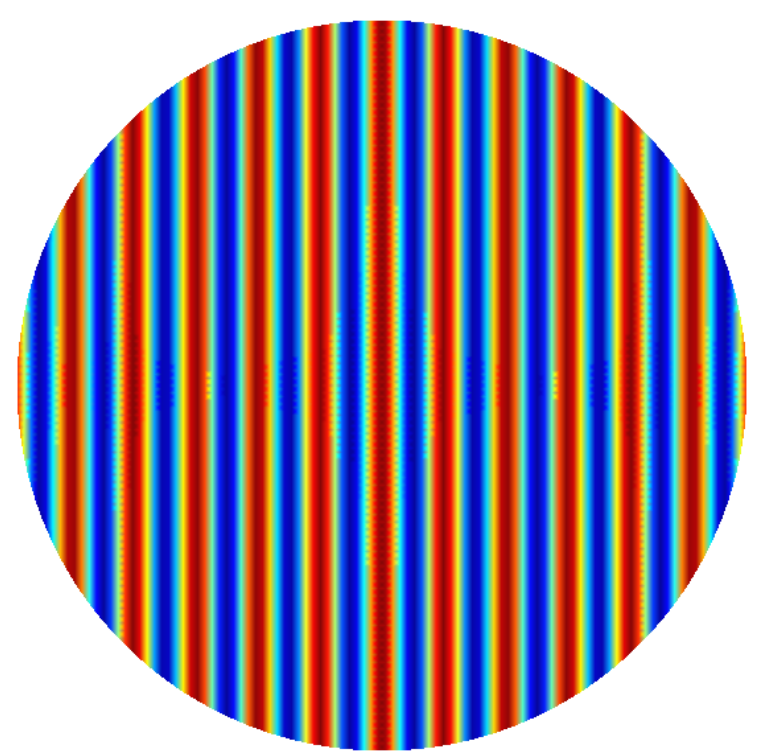

Figure 9: Solution at $2000 \mathrm{~Hz}$ for 100 subdomains

matrices on the boundaries but no new matrix in the domains. Subsequent research should focus on the improvement of the boundary condition $\frac{\partial p}{\partial n}=i k p-\frac{i}{4 k} \frac{\partial^{2} p}{\partial \tau^{2}}$.

\section{REFERENCES}

[1] C. Farhat, F.X. Roux, An unconventional domain decomposition method for an efficient parallel solution of large-scale finite element systems, SIAM J. Sci. Stat. Comput., 13, 37996, 1992.

[2] C. Farhat, P. Avery, R. Tezaur, J. Li, FETI-DPH: A dual-primal domain decomposition method for acoustic scattering, Journal of Computational Acoustics, 13, 499-524, 2005

[3] M.J. Gander, H. Zhang, Optimized Schwarz Methods with Overlap for the Helmholtz Equation, SIAM Journal on Scientific Computing, 38, No. 5, A3195-A3219, 2016.

[4] M. Gander, Optimized Schwarz methods, SIAM J. Numer. Anal., 44, 699731, 2006.

[5] M. Gander, F. Magoules, F. Natar, Optimized Schwarz methods without overlap for the Helmholtz equation, SIAM J. Sci. Comput., 24 38-60, 2002.

[6] J.-C. Nédélec, Acoustic and electromagnetic equations, in: Applied Mathematical Sciences, vol. 144, Springer-Verlag, New York, 2001, Integral representations for harmonic problems.

[7] F. Nataf, Interface connections in domain decomposition methods, In Modern methods in scientific computing and applications (Montréal, QC, 2001), volume 75 of NATO Sci. Ser. II Math. Phys. Chem., pages 323364. KluwerAcad. Publ., Dordrecht, 2002.

[8] B. Thierry, A. Vion, S. Tournier, M. El Bouajaji, D. Colignon, N. Marsic, X. Antoine, C. Geuzaine, GetDDM: An open framework for testing optimized Schwarz methods for time-harmonic wave problems, Computer Physics Communications, 203, 309-330, 2016 
[9] Y. Boubendir, X. Antoine, C. Geuzaine, A quasi-optimal non-overlapping domain decomposition algorithm for the Helmholtz equation, Journal of Computational Physics, 231, 262280, 2012.

[10] C.C. Stolk, A rapidly converging domain decomposition method for the Helmholtz equation, Journal of Computational Physics, 241, 240-252, 2013.

[11] A. Vion, C. Geuzaine, Double sweep preconditioner for optimized Schwarz methods applied to the Helmholtz problem, Journal of Computational Physics, 266, 171190, 2014.

[12] M. Eslaminia, M.N. Guddati, A double-sweeping preconditioner for the Helmholtz equation, Journal of Computational Physics, 314, 800823, 2016.

[13] A. Vion, R. Bélanger-Rioux, L. Demanet, C. Geuzaine, A DDM double sweep preconditioner for the Helmholtz equation with matrix probing of the DtN map, Proceedings of the 11th International Conference on Mathematical and Numerical Aspects of Waves (WAVES 2013), Tunis, Tunisia, June 3-7, 2013. 